\title{
FAST KVP-SWITCHING DUAL ENERGY CT FOR PET ATTENUATION CORRECTION
}

\author{
Wonseok Huh ${ }^{\dagger}$, Jeffrey A. Fessler ${ }^{\dagger}$, Adam M. Alessio $\ddagger$, and Paul E. Kinahan \\ ${ }^{\dagger}$ Department of Electrical Engineering and Computer Science, The University of Michigan. \\ ${ }^{\ddagger}$ Department of Radiology, University of Washington.
}

\begin{abstract}
$\mathrm{X}$-ray CT images are used routinely for attenuation correction in PET/CT systems. However, conventional CT-based attenuation correction (CTAC) can be inaccurate in regions containing iodine contrast agent. Dual-energy (DE) CT has the potential to improve the accuracy of attenuation correction in PET, but conventional DECT can suffer from motion artifacts. Recent X-ray CT systems can collect DE sinograms by rapidly switching the X-ray tube voltage between two levels for alternate projection views, reducing motion artifacts. The goal of this work is to study statistical methods for image reconstruction from both fast $\mathrm{kVp}$-switching $\mathrm{DE}$ scans and from conventional dual-rotate DE scans in the context of CTAC for PET.
\end{abstract}

Keywords: model-based image reconstruction, dualenergy X-ray computed tomography, penalized weighted least squares method, attenuation correction factors.

\section{INTRODUCTION}

CT-based attenuation correction (CTAC) for PET has advantages over conventional attenuation correction by PET transmission scans, including better spatial resolution and lower noise. However, PET attenuation correction factors (ACFs) must be computed for $511 \mathrm{keV}$ photon energy, whereas X-ray CT spectra cover a wide range of lower photon energies. These spectral differences can lead to imperfect PET attenuation correction, particularly when iodinated contrast agents are present [1], [2]. Conventional CTAC approaches use a single $\mathrm{X}$-ray source voltage $(\mathrm{kVp})$ and its corresponding spectrum. DECT imaging methods have the potential to improve attenuation correction in PET by exploiting the spectral information provided by using two different X-ray spectra. We previously investigated a statistically motivated, sinogram-domain approach for estimating the line integrals of two material components from DECT scans followed by computing the $511 \mathrm{keV}$ ACFs [3], [4]. That approach is iterative, but is relatively fast because the iterations are solely in the sinogram domain.

A disadvantage of sinogram-domain approaches to DECT decomposition is that the types of regularization that are suitable are limited. In contrast, by iteratively reconstructing

This work was supported in part by NIH/NCI grant 1R01CA115870. images from the measured sinograms, one can apply a wide variety of sophisticated regularizers because there can be clear distinctions between different tissue types in the images. In the sinogram domain, region boundaries are barely visible, and other forms of prior information about the object's attenuation properties are not readily apparent. Therefore, in this work we begin to explore the use of statistical methods for reconstructing material component images from DE sinogram data [5] for the purpose of PET attenuation correction.

In the field of X-ray $\mathrm{CT}$, there is increasing interest in enhancing the information provided in the images through dual-energy imaging. Dual energy (DE) CT imaging was first proposed over 30 years ago [6], but only recently became available for routine use in clinical CT systems. Various technological advances have brought renewed interest in DE CT, such as CT systems with two X-ray sources and photon counting detectors with energy selectivity. Very recently, commercial systems with fast $\mathrm{kVp}$ switching have become available, extending an idea that previously existed only in prototype systems [7]. This paper describes a model-based approach to DE image reconstruction for such systems.

The conventional approach to DE CT imaging is the "dual rotate" mode where the source is rotated around the patient at one source voltage setting to collect a full sinogram, and then the source voltage is changed (as quickly as the hardware permits) and the source is rotated around again with the new $\mathrm{kVp}$ to collect a second full sinogram. Using these two full sinograms, one can reconstruct separate images of two material components (such as soft tissue and bone) using sinogram material decomposition followed by FBP image reconstruction [6]. Model-based image reconstruction methods for fully sampled sinograms have also been proposed under monoenergetic [8], [9] and polyenergetic models [5], [10]. A drawback of this conventional "dual rotate" mode of DE CT is that the object may move between the two acquisitions, leading to inconsistencies between the two sinograms that can manifest as severe artifacts in the reconstructed images.

To reduce motion effects, the "fast $\mathrm{kVp}$ switching" mode alternates between high and low X-ray source tube voltages for the projection views. This allows DE data to be collected in a single rotation, so the motion artifacts should be comparable to those of conventional CT imaging. Modern 
CT systems rotate in less than 0.5 seconds, and collect about 1000 projection views, so the X-ray tube high voltage must be switched at $\mathrm{kHz}$ rates repeatably, which is a challenge that has been met only recently. In this switching mode, two sinograms are collected with only half as many projection views as could be collected otherwise. Conventional sinogram-domain DE decomposition methods require each ray to be measured twice by two different spectra (two different tube voltages), but in the fast switching mode, the even projection views are at one voltage and the odd views are at the other voltage.

The typical solution to this problem in fast switching DE imaging is to interpolate both sinograms in the angular direction to fill in the "missing" views. Then one can apply conventional DE decomposition followed by FBP reconstruction. However, such interpolation might compromise spatial resolution. Furthermore, DE decomposition is a noise-amplifying process, so statistical image reconstruction methods have the potential to improve image quality significantly relative to FBP in DE imaging [5]. This paper proposes a model-based image reconstruction method for DE CT that reconstructs the two material component images (e.g., soft tissue and bone) directly from the under-sampled sinograms without any interpolation. We report preliminary simulation results suggesting that this iterative method has the potential to improve image quality compared to the conventional interpolate/FBP approach.

Although current generation PET/CT scanners do not have the fast $-\mathrm{kVp}$ switching mode, it is conceivable that future PET/CT scanners will have this capability. Furthermore, the general approach proposed here is applicable not only to fast- $\mathrm{kVp}$ switching scans, but also to other DECT modes where there is "mismatch" between the rays in the highand low-energy sinograms. For example, in a dual source system with different radial sampling patterns, or in a dualhelix scan where the high-kVp and low- $\mathrm{kVp}$ helical source trajectories differ.

We have previously investigated both penalize-likelihood and penalized weighted least squares (PWLS) methods for DECT reconstruction. In this work we focus on a PWLS method that estimates two material components from undersampled sinograms (without using any interpolation). We then combine those material component images estimates at $511 \mathrm{keV}$ and reproject the PET resolution and to form ACFs. By using suitable regularization methods applied to the basis material density images, the proposed method has the potential to improve ACF accuracy compared to previous sinogram-domain approaches [4], [11].

\section{DUAL-ENERGY RECONSTRUCTION}

\section{II-A. Dual-energy CT model}

Let $y_{m i}$ denote the CT measurement for the $i$ th ray for the $m$ th incident spectrum, $m=1, \ldots, M_{0}, i=1, \ldots, N_{\mathrm{d}}$. We assume that the measurements are random variables with the following ensemble means:

$$
\bar{y}_{m i} \triangleq \int I_{m i}(\mathcal{E}) \exp \left(-\int_{\mathcal{L}_{i}} \mu(\vec{x}, \mathcal{E}) \mathrm{d} \ell\right) \mathrm{d} \mathcal{E}+r_{m i},
$$

where $\int_{\mathcal{L}_{i}} \cdot \mathrm{d} \ell$ denotes the line integral along the $i$ th ray, $I_{m i}(\mathcal{E})$ denotes the product of the $m$ th incident source spectrum, the detector gain for the $i$ th ray, $r_{m i}$ denotes additive background contributions, and $\mu(\vec{x}, \mathcal{E})$ denotes the linear attenuation coefficient (LAC) of the object being scanned at the spatial location $\vec{x}$.

We assume LAC can be represented using basis functions that are separable in the spatial and energy dimension as follows [6]:

$$
\mu(\vec{x}, \mathcal{E})=\sum_{l=1}^{L_{0}} \sum_{j=1}^{N_{\mathrm{p}}} \beta_{l}(\mathcal{E}) b_{j}(\vec{x}) \rho_{l j},
$$

where $\beta_{l}(\mathcal{E})$ denotes the energy-dependent mass-attenuation coefficient (MAC) of the $l$ th material type, $\left\{b_{j}(\vec{x})\right\}$ are (unitless) spatial basis functions such as square pixels, and $\rho_{l}=\left(\rho_{l 1}, \ldots, \rho_{l N_{\mathrm{p}}}\right)$ denotes the vector of unknown density values of $l$ th material type for each of the $N_{\mathrm{p}}$ voxels. Using (1), (2), we rewrite the ensemble means of the measurements as follows:

$$
\begin{aligned}
& \bar{y}_{m i}(\rho)=I_{m i} \mathrm{e}^{-f_{m i}(\rho)}+r_{m i} \\
& f_{m i}(\rho) \triangleq-\log \left(\int p_{m i}(\mathcal{E}) \mathrm{e}^{-\sum_{l=1}^{L_{0}} \beta_{l}(\mathcal{E}) \cdot\left[A \rho_{l}\right]_{i}} \mathrm{~d} \mathcal{E}\right),
\end{aligned}
$$

where $I_{m i}=\int I_{m i}(\mathcal{E}) \mathrm{d} \mathcal{E}$ denotes the total intensity for the $m$ th incident spectrum and the $i$ th ray, $p_{m i}(\mathcal{E}) \triangleq$ $I_{m i}(\mathcal{E}) / I_{m i}, A$ denotes the $N_{d} \times N_{p}$ system matrix having elements,

$$
a_{i j} \triangleq \int_{\mathcal{L}_{i}} b_{j}(\vec{x}) \mathrm{d} \ell .
$$

\section{II-B. Conventional Interpolation/FBP approach}

Most conventional approaches to sinogram-domain DE CT imaging have estimated the object, $\left\{\rho_{l}\right\}_{l=1}^{L_{o}}$, from fully sampled measurements, $\left\{y_{m i}\right\}_{i=1}^{N_{\mathrm{d}}}$. For fast kVp switching mode, not all sinogram rows are measured, so sinogramdomain methods require interpolation.

The "interpolation/FBP method" reconstructs the object from under-sampled sinograms. Using two steps, the interpolation/FBP method estimates $\left\{\rho_{l}\right\}_{l=1}^{L_{0}}$ from $\left\{y_{m i}\right\}_{i \in \mathcal{I}_{m}}$, where the sets of indices $\left\{\mathcal{I}_{m}\right\}_{m=1}^{M_{0}}$ are a partition of whole index set $I=\left\{1, \ldots, N_{\mathrm{d}}\right\}$. The usual estimate of $f_{m i}$ is to invert (3):

$$
\hat{f}_{m i} \triangleq-\log \left(\operatorname{smooth}\left\{\frac{Y_{m i}-r_{m i}}{I_{m i}}\right\}\right), \text { for } i \in \mathcal{I}_{m},
$$

where radial smoothing is often included to reduce noise, e.g., [12]. Using angular interpolation, one can estimate $\hat{f}_{m i}$ for all $i=1,2, \ldots, N_{\mathrm{d}}$. Then one applies conventional DE decomposition [6], followed by FBP reconstruction. This approach is fast but suboptimal. 


\section{II-C. Penalized Weighted Least Square (PWLS) ap- proach}

We propose to estimate the component density images $\rho$ directly by including a spatial roughness penalty $\mathrm{R}(\rho)$ in the following PWLS cost function:

$$
\begin{gathered}
\hat{\rho}=\underset{\rho \geq 0}{\arg \min } \Psi(\rho) \\
\Psi(\rho) \triangleq \sum_{m=1}^{M_{0}} \sum_{i \in \mathcal{I}_{m}} \frac{w_{m i}}{2}\left(\hat{f}_{m i}-f_{m i}(\rho)\right)^{2}+R(\rho),
\end{gathered}
$$

where $w_{m i}$ denotes weights that we define as follows:

$$
w_{m i}=Y_{m i}, \text { for } i \in \mathcal{I}_{m} \text {. }
$$

These weights are a reasonable choice since CT measurements are approximately Poisson distributed and in the absence of smoothing the approximate variance of $\hat{f}_{m i}$ is

$$
\operatorname{Var}\left(\hat{f}_{m i}\right) \approx \frac{\operatorname{Var}\left(Y_{m i}\right)}{\left(\bar{y}_{m i}-r_{m i}\right)^{2}} .
$$

Note that (8) uses only the measured rays $\left(i \in \mathcal{I}_{m}\right)$; no interpolation is used. The regularizing penalty term in (8) is given by the following:

$$
R(\rho)=\sum_{l=1}^{L_{0}} \sum_{j=1}^{N_{\mathrm{p}}} \sum_{k \in \mathcal{N}_{j}} \psi\left(\rho_{l j}-\rho_{l k}\right),
$$

where $\psi$ is a potential function and $\mathcal{N}_{j}$ is a neighborhood of pixel $j$. We used the modified regularization in [13] to attempt to provide approximately uniform spatial resolution. For $\psi$ we used an edge-preserving hyperbola [14] $\psi(t)=$ $\sqrt{1+(t / \delta)^{2}}$, where $\delta=0.1 \mathrm{~g} / \mathrm{cm}^{3}$.

We minimized the cost function in (8) using 100 iterations of a conjugate graduate method with a monotone line search technique [15]. We initialized the iterations using the object estimated by the interpolation/FBP method.

\section{ATTENUATION CORRECTION FACTORS}

We assume PET measurements have independent Poisson distribution:

$$
Y_{i} \sim \operatorname{Poisson}\left\{\bar{Y}_{i}(\lambda)\right\}, i=1, \ldots, N_{r},
$$

where $N_{r}$ denotes the number of detector pairs, and $\bar{Y}_{i}$ denotes the mean of the $i$ th measurement:

$$
\bar{Y}_{i}(\lambda)=\sum_{j} p_{i} a_{i j} \lambda_{j}+s_{i}
$$

where the survival probability is

$$
p_{i}=\exp \left(-\int_{L_{i}} \mu(x ; 511) \mathrm{dx}\right) .
$$

The system matrix is $a_{i j}$ and scatter and randoms are $s_{i} . \lambda_{j}$ denotes the distribution of the radio-isotope.

For reconstructing the PET emission images, one must compensate for the attenuation of annihilated photons at the
PET energy $(511 \mathrm{keV})$. In CTAC, the ACF for the $i$ th ray is defined as follows:

$$
\left.\mathrm{ACF}_{i} \triangleq \exp \left(\sum_{l=1}^{L_{0}} \beta_{l}(\mathcal{E})\left[A \hat{\rho}_{l}\right]_{i}\right)\right|_{\mathcal{E}=511 \mathrm{keV}},
$$

where $\hat{\rho}_{l}$ is an estimated density map corresponding to the $l$ th material.

In this work, we focused on the error in the PET images due to imperfections in the ACFs, so we generated noiseless PET data $\bar{Y}_{i}$ using (12 with no scatter or randoms $\left(s_{i}=\right.$ $0)$. We then multiplied the PET sinograms by the estimated ACFs (13) and applied the FBP method to reconstruct PET emission images.

We investigated four choices for the material density images $\rho$ : i) The true density maps $\rho$; ii) density map estimates $\hat{\rho}$ formed by the "interpolate/FBP" approach, from fast-kVp switching DE sinograms; iii) density map estimates $\hat{\rho}$ formed from fully sampled DE sinograms with sinogram-domain decomposition followed by FBP reconstruction, called the "FBP method"; iv) density map estimates $\hat{\rho}$ reconstructed by the iterative PWLS method.

\section{RESULTS}

We performed a preliminary examination of the proposed methods using a DECT phantom study and a simulation of DE-based CTAC for PET.

\section{IV-A. Phantom}

We applied the proposed method to dual-energy CT scans of a phantom. The sinogram data was fully sampled so we downsampled the high- and low-energy sinograms to emulate a fast-kVp switching scan. (This emulation is imperfect because it disregards the finite rise and fall times of the tube $\mathrm{kVp}$ [16], [17] that can degrade spectral separation in an actual fast-kVp scan.) The rotation center is $54.1 \mathrm{~cm}$ from the source, and the detector is $94.9 \mathrm{~cm}$ form the source. The projection space was 888 radial samples $\times 820$ angular views and The reconstructed images were $512 \times 512$ with $0.1 \times 0.1 \mathrm{~cm}^{2}$ pixel size. We applied the conventional FBP reconstruction method, dual-energy interpolation/FBP reconstruction method, and the proposed regularized PWLS method with source voltages $80 \mathrm{kVp}$ and $140 \mathrm{kVp}$. This phantom scan was a low dose, $10 \mathrm{~mA}$, study.

Fig. 1 shows the component material images reconstructed by the three methods. Fig. 1(a)-(b) shows the conventional (fully sampled) FBP images, whereas Fig. 1(c)-(d) shows interpolation/FBP method from emulated fast-kVp switching views. Both methods' results are noisy and have many streaks due to the low dose of this CT data. However, the PWLS images, in Fig. 1(e)-(f), have successfully reduced streak artifacts and yield lower noise than other two methods even though it also the used under-sampled CT data. For completeness, we also applied the proposed method to 


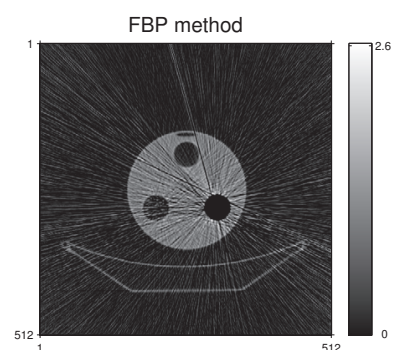

(a) Soft tissues

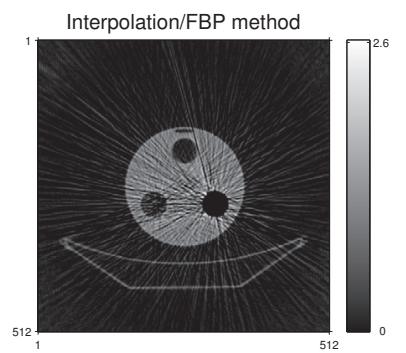

(c) Soft tissues

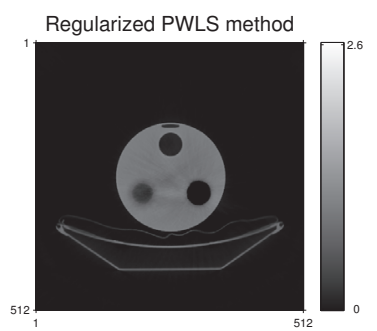

(e) Soft tissues

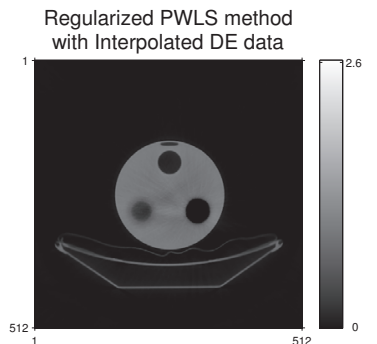

(g) Soft tissues

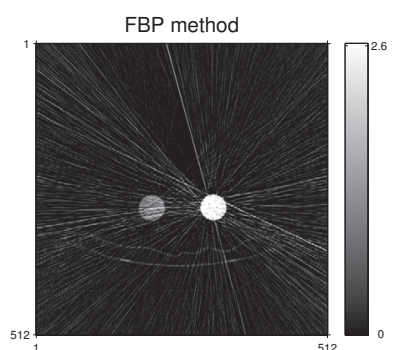

(b) Bone minerals

Interpolation/FBP method

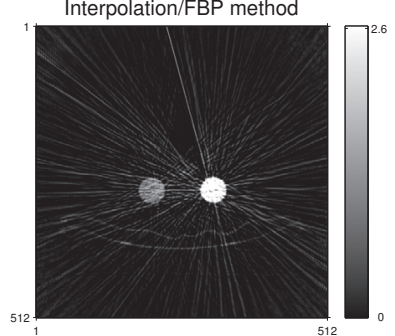

(d) Bone minerals

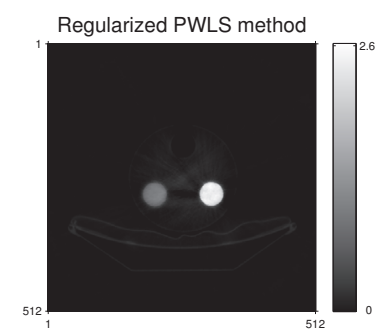

(f) Bone minerals

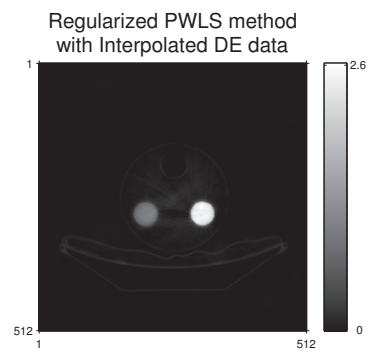

(h) Bone minerals
Fig. 1. First row: FBP method, Second row: Interpolation/FBP method, Third row: PWLS method, Fourth row: PWLS method from interpolated DE data.

the interpolated DE data. Fig. $1(\mathrm{~g})-(\mathrm{h})$ shows the resulting images. As shown in Fig. 1, the proposed method has the almost the performance is very similar to Fig. 1(e) and (f).

\section{IV-B. Simulation}

To evaluate the performance of the proposed method for CTAC, we compared it to two traditional methods: the (fully sampled) FBP method and the interpolation/FBP method. We applied three methods to simulated data with the same parameters in real phantom data.

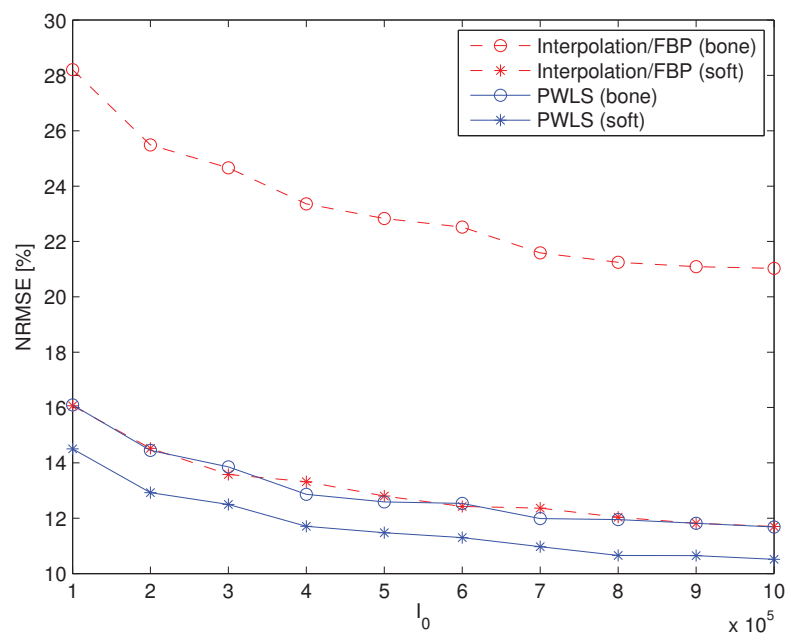

Fig. 2. NRMSE of estimated density maps: Interpolation/FBP method, and the proposed method.

Fig. 2 shows the NRMSE plot of estimated density maps with different incident intensities, $I_{0}$. We observed that the proposed method significantly reduces the NRMSE of soft tissue and bone minerals compared to the interpolation/FBP method.

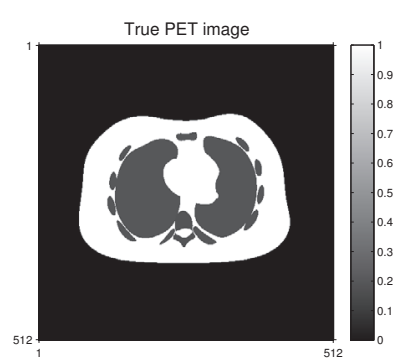

(a) PET image, ACF by true CT density map

Interpolation/FBP method

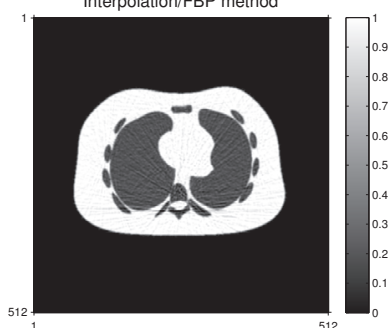

(c) PET image, ACF

by Interpolation/FBP method

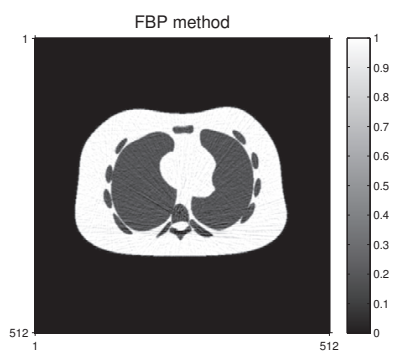

(b) PET image, ACF by FBP method

Regularized PWLS method

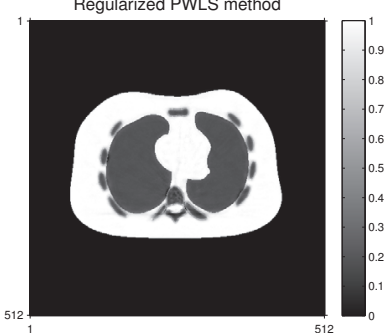

(d) PET image, ACF

by Regularized PWLS method
Fig. 3. Reconstructed PET emission images using competing attenuation correction methods.

Fig. 3 shows the (FBP) reconstructed PET emission images based on the various methods for estimating the ACFs. The PET images in Fig. 3(d) are based on ACFs from 
the PWLS DE reconstruction method. This CTAC approach provides reduced noise and streak artifacts compared to the other methods in Fig. 3(b)-(c).

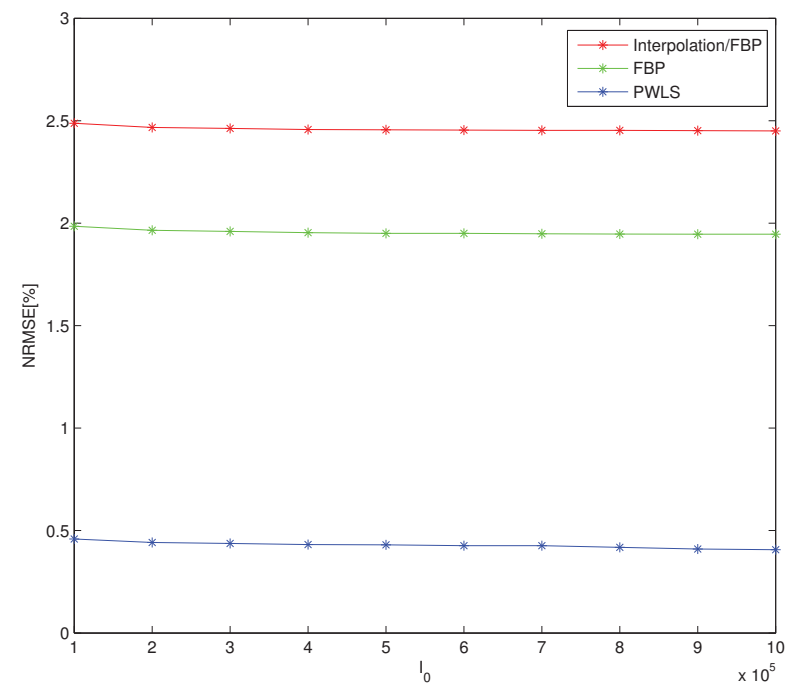

Fig. 4. NRMSE of reconstructed PET images: Interpolation/FBP method, and the proposed method, versus $I_{0}$ (number of incident $\mathrm{X}$-ray photons per ray).

Fig. 4 compares the NRMSE of the reconstructed PET images. The NRMSE of the PET image based on the PWLS ACFs is significantly lower than the other competing methods investigated here.

\section{DISCUSSION}

We presented an iterative PWLS algorithm for DE CT reconstruction, motivated by improving attenuation correction in PET. The regularized method estimates the two material component images directly from DE sinograms including the type of under-sampled DE data that is collected by fast $\mathrm{kVp}$ switching CT systems. Unlike other DE CT algorithms, the proposed method estimates material component images directly from only half as many projection views without any interpolation operation. Using these estimated DE CT images yields more accurate ACFs than conventional approaches.

Our next step is to evaluate the method with real patient data and to investigate penalized-likelihood methods. Additionally, we observed undesirable variations in spatial resolution and cross talk between the soft tissue and bone material images. We need to analyze the spatial resolution and noise properties of statistical methods for DE reconstruction and use this analysis to improve the design of the regularizers. This problem is relatively challenging because of the nonlinearities of beam hardening in DECT with polyenergetic spectra.

\section{REFERENCES}

[1] P. E. Kinahan, A. M. Alessio, and J. A. Fessler, "Dual energy CT attenuation correction methods for quantitative assessment of response to cancer therapy with PET/CT imaging," Technology in Cancer Research and Treatment, vol. 5, no. 4, pp. 319-28, Aug. 2006.

[2] M. Shirmohammad, M. R. Ay, S. Sarkar, H. Ghadiri, and A. Rahmim, "Comparative assessment of different energy mapping methods for generation of 511-keV attenuation map from CT images in PET/CT systems: A phantom study," in Proc. IEEE Intl. Symp. Biomed. Imag., 2008, pp. 644-7.

[3] J. Noh, J. A. Fessler, and P. E. Kinahan, "Low-dose dualenergy computed tomography for PET attenuation correction with statistical sinogram restoration," in Proc. SPIE 6913, Medical Imaging 2008: Phys. Med. Im., 2008, pp. 691312:110.

[4] J. Noh, J. A. Fessler, and P. E. Kinahan, "Statistical sinogram restoration in dual-energy CT for PET attenuation correction," IEEE Trans. Med. Imag., vol. 28, no. 11, pp. 1688-702, Nov. 2009.

[5] J. A. Fessler, I. Elbakri, P. Sukovic, and N. H. Clinthorne, "Maximum-likelihood dual-energy tomographic image reconstruction," in Proc. SPIE 4684, Medical Imaging 2002: Image Proc., 2002, vol. 1, pp. 38-49.

[6] R. E. Alvarez and A. Macovski, "Energy-selective reconstructions in X-ray computed tomography," Phys. Med. Biol., vol. 21, no. 5, pp. 733-44, Sept. 1976.

[7] W. A. Kalender, W. H. perman, J. R. Vetter, and E. Klotz, "Evaluation of a prototype dual-energy computed tomographic apparatus. 1. Phantom studies," Med. Phys., vol. 13, no. 3, pp. 334-9, May 1986.

[8] N. H. Clinthorne, "A constrained dual-energy reconstruction method for material-selective transmission tomography," Nucl. Instr. Meth. Phys. Res. A., vol. 353, no. 1, pp. 347-8, Dec. 1994.

[9] P. Sukovic and N. H. Clinthorne, "Penalized weighted leastsquares image reconstruction for dual energy X-ray transmission tomography," IEEE Trans. Med. Imag., vol. 19, no. 11, pp. 1075-81, Nov. 2000.

[10] J. A. O'Sullivan and J. Benac, "Alternating minimization algorithms for transmission tomography," IEEE Trans. Med. Imag., vol. 26, no. 3, pp. 83-297, Mar. 2007.

[11] P. J. La Riviere and P. Vargas, "Penalized-likelihood sinogram decomposition for dual-energy computed tomography," in Proc. IEEE Nuc. Sci. Symp. Med. Im. Conf., 2008, pp. 5166-9.

[12] J. Hsieh, "Adaptive streak artifact reduction in computed tomography resulting from excessive x-ray photon noise," Med. Phys., vol. 25, no. 11, pp. 2139-47, Nov. 1998.

[13] J. A. Fessler and W. L. Rogers, "Spatial resolution properties of penalized-likelihood image reconstruction methods: Spaceinvariant tomographs," IEEE Trans. Im. Proc., vol. 5, no. 9, pp. 1346-58, Sept. 1996.

[14] P. Charbonnier, L. Blanc-Féraud, G. Aubert, and M. Barlaud, "Two deterministic half-quadratic regularization algorithms for computed imaging," in Proc. IEEE Intl. Conf. on Image Processing, 1994, vol. 2, pp. 168-71.

[15] J. A. Fessler and S. D. Booth, "Conjugate-gradient preconditioning methods for shift-variant PET image reconstruction," IEEE Trans. Im. Proc., vol. 8, no. 5, pp. 688-99, May 1999.

[16] Y. Zou and M. D. Silver, "Analysis of fast kV-switching in dual energy CT using a pre-reconstruction decomposition technique," in Proc. SPIE 6913, Medical Imaging 2008: Phys. Med. Im., 2008, pp. 691313-12.

[17] D. Xu, D. A. Langan, X. Wu, J. D. Pack, T. M. Benson, J. E. 
Tkaczky, and A. M. Schmitz, "Dual energy CT via fast kVp switching spectrum estimation," in Proc. SPIE 7258, Medical Imaging 2009: Phys. Med. Im., 2009, p. 72583T. 